\title{
Mortalidade materna em Teresina, Piauí, Brasil: um estudo caso-controle
}

\section{Maternal mortality in Teresina, Piauí, Brazil: a case-control study}

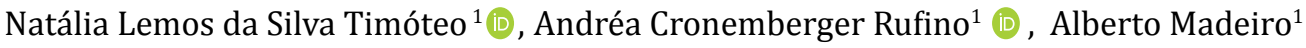

1. Programa de Pós-Graduação em Saúde e Comunidade, Universidade Federal do Piauí (UFPI), Teresina, Piauí, Brasil

\section{Resumo}

\begin{abstract}
Objetivo: Analisar os óbitos maternos ocorridos entre 2012 e 2016 em Teresina, Piauí, Brasil. Métodos: Realizou-se estudo caso-controle, cujos casos corresponderam aos óbitos maternos cujos controles foram constituídos por mulheres que tiveram parto no mesmo período, mas não morreram. Foram empregadas análises uni e multivariada. Resultados: A razão de mortalidade materna global foi de 65,7/100.000 nascidos vivos. Ocorreu predomínio das causas obstétricas diretas (69,4\%), sendo aborto (25\%) e doenças hipertensivas $(19,4 \%)$ as mais frequentes. Mulheres com idade $\geq 30$ anos, sem ocupação e aquelas que não realizaram pré-natal ou até três consultas apresentaram maior chance de óbito materno. No modelo multivariado, apenas o baixo número de consultas pré-natais (ORaj=4,33; IC95\% 3,01-7,34) se mostrou associado à morte materna. Conclusões: Houve destaque para causas evitáveis de morte, com chance de óbito maior entre mulheres com atenção pré-natal inadequada. É necessário ter melhor qualificação do planejamento familiar, pré-natal e assistência ao parto e puerpério no município.
\end{abstract}

Palavras-chave: Mortalidade Materna; Estudos de Casos e Controles; Sistemas de Informação; Fatores de Risco. Saúde Reprodutiva.

\begin{abstract}
Objective: To analyze the maternal deaths that occurred between 2012 and 2016 in Teresina, Piauí, Brazil. Methods: We conducted a case-control study, whose cases corresponded to maternal deaths whose controls consisted of women who gave birth in the same period but did not die. Univariate and multivariate analyzes were employed. Results: The overall maternal mortality ratio was 65.7/ 100,000 live births. There was a predominance of direct obstetric causes (69.4\%), with abortion (25\%) and hypertensive diseases (19.4\%) being the most frequent. Women aged $\geq 30$ years, without occupation, and those who did not receive prenatal care or up to three consultations had a higher chance of maternal death. In the multivariate model, only the low number of prenatal consultations (ORad=4.33; 95\%IC 3.01-7.34) was associated with maternal death. Conclusions: Avoidable causes of death were highlighted, with a higher chance of death among women with inadequate prenatal care. It is necessary to have better qualification of family planning, prenatal and childbirth and postpartum care in the municipality.
\end{abstract}

Keywords: Maternal Mortality; Case-control Studies; Information Systems; Risk Factors; Reproductive Health.

INTRODUÇÃO

A morte materna ainda persiste como um grande desafio para a saúde pública. Houve redução de $44 \%$ da razão de mortalidade materna (RMM) no mundo, nos últimos 25 anos, tendo a estimativa declinado de 385 óbitos maternos por 100.000 nascidos vivos (NV), em 1990, para 216 óbitos maternos por 100.000 NV em 2015. Porém, as estimativas globais da mortalidade materna (MM) mostraram que os resultados foram insuficientes para alcançar, na maioria dos países, o 50 Objetivo de Desenvolvimento do Milênio (ODM), de reduzir em $75 \%$ a RMM em 15 anos $^{1}$. Em 2015, cerca de 300.000 mulheres morreram durante e após a gravidez/ parto, e a maioria dos óbitos é por causas evitáveis e/ou tratáveis².
No mundo, $99 \%$ das mortes maternas ocorrem em países em desenvolvimento. Mais da metade desses óbitos são observados na África subsaariana, e quase um terço deles no sul da Ásia. O risco de uma mulher morrer por causas relacionadas à gravidez, ao parto ou ao puerpério varia de 1 para cada 150 nas regiões em desenvolvimento a 1 para cada 4.900 nas regiões desenvolvidas. A RMM, em países em desenvolvimento em 2015, foi de 239 por 100.000 NV comparada a 12 por $100.000 \mathrm{NV}$ em países desenvolvidos ${ }^{1,2}$. Tais fatos evidenciam as disparidades existentes entre os países, ainda ressaltando que o risco de mortalidade materna é maior para mulheres que vivem em áreas rurais e em comunidades mais pobres. 
As principais causas de mortalidade materna são hemorragias, distúrbios hipertensivos, sepse e complicações relacionadas ao aborto, mas com importantes variações geográficas. As hemorragias representaram $36,9 \%$ de óbitos no norte da África, mas apenas $16,3 \%$ nas regiões desenvolvidas. Por sua vez, os distúrbios hipertensivos foram uma causa, particularmente, importante de morte na América Latina e no Caribe, contribuindo com $22,1 \%$ de todas as mortes maternas na região. Quase todas as mortes por sepse foram registradas nos países em desenvolvimento, e a proporção dessas mortes foi maior no sul da Ásia $(13,7 \%)^{3}$. Grande parte dos óbitos maternos é reflexo da qualidade de assistência durante o ciclo gravídico-puerperal, tendo a idade materna elevada, a baixa escolaridade e o número reduzido de consultas pré-natais como fatores associados bem conhecidos ${ }^{4,5}$.

No cenário brasileiro, ocorreu uma redução da RMM nas últimas décadas. A RMM passou de 141 óbitos maternos por 100.000 NV em 1990 para 68 mortes maternas por 100.000 NV em $2010^{6}$. Entretanto, a redução não foi suficiente para que o país atingisse a meta estabelecida pelo 50 ODM, que era chegar a uma taxa de 35 mortes por $100.000 \mathrm{NV}^{7}$. Entre os anos de 1990 e 2010, as duas principais causas específicas de morte materna foram a hipertensão e a hemorragia, seguidas pela infecção puerperal e as complicações relacionadas ao aborto. Entre as causas indiretas, o grupo com maior frequência foi o das doenças do aparelho circulatório complicadas pela gestação, pelo parto ou pelo puerpério ${ }^{8}$.

Em termos regionais, Norte e Nordeste apresentam índices maiores que Sul, Sudeste e Centro-Oeste. Entre 2009 e 2011, por exemplo, a região Nordeste registrou RMM de 80,8 óbitos maternos por $100.000 \mathrm{NV}$, enquanto a região Sul apresentou RMM de 54,5 óbitos maternos por 100.000 NV. Dados de 2009-2011 evidenciam que o estado do Piauí apresentou a 2a maior RMM $(101,8 / 100.000 \mathrm{NV})$ do país, ficando atrás somente do Maranhão (114/100.000 NV) ${ }^{9}$. Por outro lado, pesquisa realizada entre os anos de 2012 e 2013 no único hospital terciário em obstetrícia do estado apontou que os principais determinantes da morbidade materna grave foram distúrbios hipertensivos $(86,1 \%)$, distúrbios hemorrágicos $(10,0 \%)$ e os distúrbios infecciosos (2,9\%). No tocante às causas de morte materna, houve destaque para causas hipertensivas (40\%), hemorrágicas $(30 \%)$ e infecciosas $(30,0 \%)^{10}$. Este estudo teve como objetivo analisar os óbitos maternos ocorridos entre 2012 e 2016 em Teresina, Piauí, descrevendo magnitude, causas básicas e fatores de riscos.

\section{MÉTODOS}

Trata-se de estudo observacional, do tipo caso-controle, realizado no período de setembro a novembro de 2019 no Núcleo de Eventos Vitais da Fundação Municipal de Saúde de Teresina.

O grupo caso compreendeu 36 óbitos maternos de mulheres residentes em Teresina, ocorridos no período de 2012 a 2016 (último ano com dados disponíveis). Para esse grupo, os critérios de inclusão foram mulheres em idade fértil (10 a 49 anos), que residiam no município de Teresina e que foram a óbito no período de 10 de janeiro de 2012 a 31 de dezembro de 2016, cujas mortes foram notificadas no Sistema de Informações de Mortalidade (SIM) e investigadas pelo Serviço de Vigilância do Óbito Materno (SVOM) da Fundação Municipal de Saúde de Teresina.

O grupo controle compreendeu 144 mulheres residentes de Teresina, que também apresentaram como critérios de inclusão engravidar e ter tido parto no mesmo período (2012-2016), porém não evoluíram ao óbito. Foram identificadas 68.748 mães de nascidos vivos no Sistema de Informações sobre Nascidos Vivos (SINASC). Houve exclusão das mulheres cujo registro não dispunha de informação para todas as variáveis analisadas. Dessa forma, restaram 60.187 mulheres no banco de dados. 0 grupo controle foi formado na razão de 4:1 em relação ao grupo caso, por meio de amostragem aleatória simples, tendo, ao final, 144 controles (figura 1).

A coleta dos dados secundários foi realizada por meio da análise de todas as declarações de óbitos maternos e das fichas de investigação do óbito materno por um dos pesquisadores, previamente treinado, e com auxílio de formulário semiestruturado. $O$ instrumento era composto de dados sociodemográficos (faixa etária, cor da pele, escolaridade, estado civil, ocupação), clínicos (número de partos, número de consultas pré-natais, tipo de gestação, período da gestação, término da gestação) e características do óbito (causa básica do óbito e classificação do óbito).

Realizou-se o cálculo da razão de mortalidade materna (número de óbitos maternos dividido pelo número de nascidos vivos e multiplicado por 100.000 , para cada ano) e também das causas básicas, por meio da causa afirmada na declaração de óbito e classificada conforme a CID-10.

Para a análise bi e multivariada, as variáveis sociodemográficas e clínicas foram dicotomizadas. Empregou-se o teste do quiquadrado ou o teste exato de Fisher (quando, pelo menos, uma das frequências esperadas foi menor do que 5) para verificar a associação entre as variáveis categóricas e o desfecho morte materna. Foram calculadas odds ratios brutas (ORbr) e ajustadas (ORaj) e seus respectivos intervalos de confiança de 95\% (IC95\%). Construiu-se modelo de regressão logística múltipla (forward stepwise selection) e, para tal, foram incluídas todas as variáveis que alcançaram $p<0,25$ na análise bivariada. Para verificar a adequação do modelo, utilizou-se o teste de Hosmer e Lemeshow. Todas as análises estatísticas foram realizadas com o software SPSS para Mac, versão 22.

A pesquisa foi autorizada pela Fundação Municipal de Saúde de Teresina e aprovada pelo Comitê de Ética em Pesquisa da Universidade Federal do Piauí, sob o Parecer no 2.763.910/ CAAE 92102418.6.0000.5214. 
Figura 1. Fluxograma da estruturação final do banco de dados. Teresina, Piauí, Brasil.

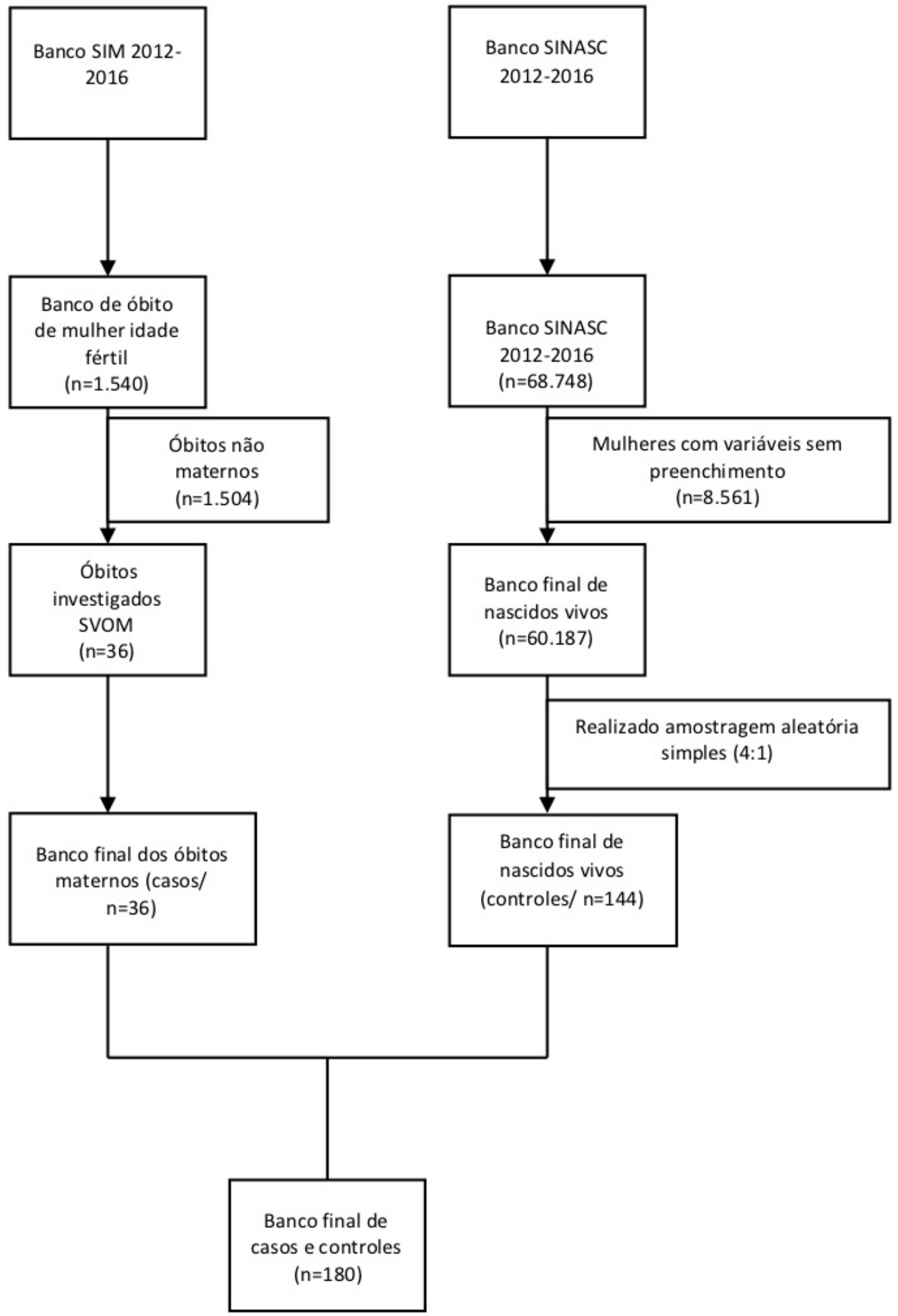




\section{RESULTADOS}

No período do estudo (2012-2016), foram notificados 36 óbitos maternos no município de Teresina. A tabela 1 evidencia que a maior parte das mulheres apresentava de 30 a 39 anos (38,9\%), era de cor parda $(66,7 \%)$, possuía 12 anos ou mais de estudo (38,9\%), tinha parceria conjugal $(47,2 \%)$ e não exercia ocupação remunerada $(44,4 \%)$. Mulheres na segunda gravidez foram mais frequentes $(50,0 \%)$, e a principal forma de término da gestação foi a cesárea (41,7\%), porém 9 óbitos $(25 \%)$ ocorreram após abortos. Chama a atenção que 30,6\% das mulheres não realizaram pré-natal.

Houve variação na razão de mortalidade materna no período analisado, sendo o maior valor (94/100.000 NV) em 2012 com 13 óbitos notificados, e a menor taxa foi registrada no ano de 2014 (21,5/100.000 NV) com 3 óbitos maternos. Entre 2012 e 2016, a RMM foi de 65/100.000 NV.

Tabela 1. Características sociodemográficas e clínicas dos óbitos maternos.

Teresina, Piauí, Brasil.

\begin{tabular}{lrr}
\hline Variáveis & $\mathbf{n}$ & $\%$ \\
\hline Faixa etária (anos) & & \\
$10-14$ & 2 & 5,6 \\
$15-19$ & 7 & 19,4 \\
$20-29$ & 11 & 30,5 \\
$30-39$ & 14 & 38,9 \\
$40-49$ & 2 & 5,6
\end{tabular}

\section{Cor da pele*}

$\begin{array}{lrr}\text { Branca } & 4 & 11,1 \\ \text { Preta } & 4 & 11,1 \\ \text { Parda } & 24 & 66,7\end{array}$

$\begin{array}{lrr}\text { Escolaridade }(\mathrm{anos})^{+} & & \\ \text {Nenhuma } & 1 & 2,8 \\ 4-7 & 5 & 13,9 \\ 8-11 & 10 & 27,8 \\ 12 & 14 & 38,9\end{array}$

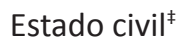

Solteira

$12 \quad 33,3$

Casada/União estável

1747,2

$\begin{array}{lll}\text { Ocupação§ } & & \\ \text { Sim } & 10 & 27,8 \\ \text { Não } & 16 & 44,4\end{array}$

Número de partos"

\begin{tabular}{lll} 
Nenhum & 11 & 30,5 \\
1 & 18 & 50,0 \\
$2-3$ & & \\
\hline
\end{tabular}

\begin{tabular}{|c|c|c|}
\hline Variáveis & $\mathrm{n}$ & $\%$ \\
\hline \multicolumn{3}{|l|}{ Tipo de gestaçãoף } \\
\hline Única & 20 & 55,5 \\
\hline Múltipla & 1 & 2,8 \\
\hline \multicolumn{3}{|l|}{ Período da gestação** } \\
\hline 10 trimestre & 4 & 11,1 \\
\hline 20 trimestre & 7 & 19,4 \\
\hline 30 trimestre & 5 & 13,9 \\
\hline Não se aplica & 18 & 50 \\
\hline \multicolumn{3}{|l|}{ Término da gestação†† } \\
\hline Vaginal & 3 & 8,3 \\
\hline Cesárea & 15 & 41,7 \\
\hline Aborto & 9 & 25,0 \\
\hline Ectópica (salpingectomia) & 1 & 2,8 \\
\hline Não se aplica & 7 & 19,4 \\
\hline \multicolumn{3}{|c|}{ Número de consultas pré-natais $\ddagger$} \\
\hline Nenhuma & 11 & 30,6 \\
\hline $1-3$ & 6 & 16,7 \\
\hline $4-6$ & 7 & 19,4 \\
\hline$\geq 7$ & 8 & 22,2 \\
\hline
\end{tabular}

*Ignorado: 04 casos $(11,1 \%)$; † Ignorado: 06 casos $(16,6 \%)$; ¥ Ignorado: 07 casos (19,5\%); §lgnorado: 10 casos; $(27,8 \%)$; || Ignorado: 01 caso (2,8\%); Ilgnorado: 15 casos $(41,7 \%)$; **Ignorado: 02 casos (5,6\%); †t|gnorado: 01 caso $(2,8 \%)$; ¥¥lgnorado: 04 casos $(11,1 \%)$.

A tabela 2 mostra que as causas obstétricas diretas responderam por $69,4 \%$ das mortes maternas, as indiretas, por $25,0 \%$ e as tardias por 5,6\%. Entre as diretas, houve predominância de complicações de abortos (25\%), de doenças hipertensivas $(19,4 \%)$, de infecções $(8,3 \%)$ e de complicações do trabalho de parto e do parto $(5,6 \%)$. Entre as causas obstétricas indiretas, prevaleceram as doenças do aparelho respiratórias $(8,3 \%)$ e digestivas (5,6\%).

Mulheres com idade igual ou superior a 30 anos apresentaram maior chance de óbito materno (ORbr=2,34; IC95\% 1,92-3,71) quando comparadas àquelas com faixa etária inferior a 30 anos. Além disso, as mulheres sem ocupação apresentaram chance mais elevada (ORbr=1,76; IC95\% 1,32-2,44) de morte materna, comparadas às mulheres com ocupação. Por fim, comparadas com grávidas que completaram 4 ou mais consultas pré-natais, gestantes que não realizaram pré-natal ou até três consultas apresentaram maior chance de mortalidade materna $(\mathrm{ORbr}=4,86$; IC95\% 2,98-5,21). As variáveis cor da pele, escolaridade e conjugalidade não apresentaram associação significativa com o desfecho de morte materna (tabela 3 ).

No modelo multivariado, apenas a atenção pré-natal se 
apresentou como fator associado à mortalidade materna. Gestantes que não realizaram pré-natal ou até três consultas mantiveram maior chance (ORaj=4,33; IC95\% 3,01-7,34) de óbito materno comparadas com grávidas com quatro ou mais consultas (tabela 4).

Tabela 2. Óbitos maternos por causas básicas, de acordo com CID-10. Teresina, Piauí, Brasil.

\begin{tabular}{|c|c|c|}
\hline Causa básica dos óbitos (CID-10) & $\mathbf{N}$ & $\%$ \\
\hline OBSTÉTRICAS DIRETAS & 25 & 69,4 \\
\hline \multicolumn{3}{|l|}{ Gravidez que termina em aborto (000-008) } \\
\hline Incompleto, complicado por infecção (008.0) & 4 & 11,1 \\
\hline Aborto não especificado (006) & 2 & 5,5 \\
\hline Outras complicações (008.8) & 1 & 2,8 \\
\hline Hemorragia tardia ou excessiva (008.1) & 1 & 2,8 \\
\hline Aborto retido (002.1) & 1 & 2,8 \\
\hline Gravidez ectópica (O00) & 1 & 2,8 \\
\hline \multicolumn{3}{|l|}{ Doenças hipertensivas (010-016) } \\
\hline Pré-eclâmpsia grave (014.1) & 6 & 16,6 \\
\hline Eclâmpsia na gravidez (015.0) & 1 & 2,8 \\
\hline Infecção puerperal (085) & 3 & 8,3 \\
\hline \multicolumn{3}{|l|}{ Complicações trabalho de parto e parto (060-075) } \\
\hline Rotura uterina (071.1) & 1 & 2,8 \\
\hline Outras formas de inércia uterina (062.2) & 1 & 2,8 \\
\hline Placenta prévia com hemorragia (044.1) & 1 & 2,8 \\
\hline Cardiomiopatia no puerpério (090.3) & 1 & 2,8 \\
\hline Embolia amniótica (088.1) & 1 & 2,8 \\
\hline OBSTÉTRICAS INDIRETAS & 9 & 25,0 \\
\hline Doenças do aparelho respiratório (099.5) & 3 & 8,3 \\
\hline \multicolumn{3}{|l|}{ Doenças do aparelho digestivo (099.6) } \\
\hline Colecistite (K81) & 1 & 2,8 \\
\hline Colangite (K83.0) & 1 & 2,8 \\
\hline Doenças do aparelho circulatório (099.4) & 1 & 2,8 \\
\hline Trombocitopenia não especificada (D69.6) & 1 & 2,8 \\
\hline Influenza devido vírus não identificado (J11.0) & 1 & 2,8 \\
\hline Outras doenças complicando a gravidez, parto e puerpério (099.8) & 1 & 2,8 \\
\hline OBSTÉTRICAS TARDIAS & 2 & 5,6 \\
\hline Pneumonia não classificada em outra parte (J15) & 1 & 2,8 \\
\hline Cardiomiopatia no puerpério (090.3) & 1 & 2,8 \\
\hline
\end{tabular}


Mortalidade materna em Teresina

Tabela 3. Análise bivariada dos fatores associados com a mortalidade materna. Teresina, Piauí, Brasil.

\begin{tabular}{|c|c|c|c|c|c|c|}
\hline \multirow[t]{2}{*}{ Variáveis } & \multicolumn{2}{|c|}{ Casos } & \multicolumn{2}{|c|}{ Controles } & \multirow[t]{2}{*}{ OR* (IC 95\%)† } & \multirow[t]{2}{*}{$\mathrm{p} \neq$} \\
\hline & $\mathbf{n}$ & $\%$ & $\mathbf{n}$ & $\%$ & & \\
\hline \multicolumn{7}{|l|}{ Faixa etária } \\
\hline$<30$ anos & 20 & 55,6 & 122 & 84,7 & 1,00 & - \\
\hline$\geq 30$ anos & 16 & 44,4 & 22 & 15,2 & $2,34(1,92-3,71)$ & 0,004 \\
\hline \multicolumn{7}{|l|}{ Cor da pele } \\
\hline Branca & 4 & 11,1 & 17 & 11,8 & 1,00 & - \\
\hline Não branca & 28 & 77,7 & 127 & 88,2 & $1,94(0,76-2,34$ & 0,266 \\
\hline \multicolumn{7}{|l|}{ Escolaridade } \\
\hline$<4$ anos & 4 & 11,1 & 15 & 10,4 & $1,45(1,03-2,88)$ & 0,078 \\
\hline$\geq 4$ anos & 26 & 72,2 & 129 & 89,6 & 1,00 & - \\
\hline \multicolumn{7}{|l|}{ Conjugalidade } \\
\hline Com parceria & 17 & 47,2 & 98 & 68,1 & 1,00 & - \\
\hline Sem parceria & 12 & 33,3 & 46 & 31,9 & $0,87(0,68-1,46)$ & 0,278 \\
\hline \multicolumn{7}{|l|}{ Ocupação } \\
\hline Com ocupação & 16 & 44,4 & 139 & 96,5 & 1,00 & - \\
\hline Sem ocupação & 10 & 27,8 & 5 & 3,5 & $1,76(1,32-2,44)$ & 0,038 \\
\hline \multicolumn{7}{|c|}{ Número de partos } \\
\hline 0 & 11 & 30,6 & 47 & 32,6 & 1,00 & - \\
\hline$\geq 1$ & 24 & 66,7 & 97 & 67,4 & $1,45(0,98-2,03)$ & 0,431 \\
\hline \multicolumn{7}{|c|}{ Término da gestação } \\
\hline Vaginal & 3 & 8,3 & 80 & 55,6 & 1,00 & - \\
\hline Cesárea & 15 & 41,7 & 64 & 44,4 & $1,87(1,11-2,98)$ & 0,329 \\
\hline \multicolumn{7}{|c|}{ Consultas pré-natais } \\
\hline $0-3$ & 17 & 47,2 & 15 & 10,4 & $4,86(2,98-5,21)$ & 0,001 \\
\hline$\geq 4$ & 15 & 41,7 & 129 & 89,6 & 1,00 & - \\
\hline
\end{tabular}

*OR: odds ratio; †IC95\%: intervalo de confiança de 95\%; ¥Teste do qui-quadrado/ teste exato de Fisher; *p <0,05

Tabela 4. Análise multivariada dos fatores preditivos para mortalidade materna.

Teresina, Piauí, Brasil.

\begin{tabular}{|c|c|c|c|c|}
\hline Variável & ORbr* & ORaj† & IC95\%‡ (ORaj) & $\mathbf{p}$ \\
\hline \multicolumn{5}{|l|}{ Faixa etária } \\
\hline$<30$ anos & - & - & $1,85-9,05$ & 0,079 \\
\hline$\geq 30$ anos & 2,34 & 2,12 & & \\
\hline \multicolumn{5}{|l|}{ Escolaridade } \\
\hline$<4$ anos & 1,45 & 1,22 & $0,88-5,43$ & 0,146 \\
\hline$\geq 4$ anos & - & - & & \\
\hline \multicolumn{5}{|l|}{ Ocupação } \\
\hline Com ocupação & - & - & $0,95-4,07$ & 0,109 \\
\hline Sem ocupação & 1,76 & 1,94 & & \\
\hline \multicolumn{5}{|c|}{ Consultas pré-natais } \\
\hline $0-3$ & 4,86 & 4,33 & $3,01-7,34$ & $0,001^{*}$ \\
\hline$\geq 4$ & - & - & & \\
\hline
\end{tabular}

Teste de Hosmer e Lemeshow: $p=0,321 ;$ *ORbr: odds ratio bruto; †ORaj: odds ratio ajustado; $¥$ IC95\%: intervalo de confiança a $95 \%$. 


\section{DISCUSSÃO}

O padrão de mortalidade materna observado neste estudo foi semelhante ao encontrado em outras capitais brasileiras ${ }^{11,12}$. O município de Teresina apresentou RMM global alta de $65,7 / 100.000 \mathrm{NV}$, conforme a categorização da $\mathrm{OMS}^{13}$. Em termos comparativos, essa RMM está um pouco abaixo da média do país $(68,2 / 100.000 \mathrm{NV})^{9}$. No entanto, observa-se que o valor da RMM é superior a outras capitais brasileiras. Como exemplo, a RMM no município de São Paulo entre 2004-2013, na região Sudeste, foi de $55,9 / 100.000$ NV $^{14}$. Na região Norte, o município de Belém-Pará, em 2004, apresentou valor de 57,3 por 100.000 $\mathrm{NV}^{15}$. Por outro lado, outras cidades brasileiras apresentaram RMMs mais elevadas que o presente estudo, como Juiz de Fora-Minas Gerais, no período de 2005 a 2015, com valor de $119,6 / 100.000 N^{16}$. Porém, é importante contextualizar que esses dados discrepantes podem ser decorrentes da maior oferta e da melhor infraestrutura dos serviços de saúde das capitais dos estados, gerando menores taxas de mortalidade.

Em relação às causas básicas dos óbitos analisadas neste estudo, observou-se maior preponderância para a mortalidade materna por causas diretas. O aborto, as doenças hipertensivas e a infecção puerperal constituíram as principais causas obstétricas diretas de óbito materno, nessa ordem. Uma pesquisa sobre a mortalidade de mulheres em idade fértil no Piauí revelou que, no período de 2008 a 2012, as doenças hipertensivas foram a principal causa de óbito obstétrico direto, seguida das desordens hemorrágicas e das complicações do aborto ${ }^{17}$. Chama a atenção para as causas hemorrágicas que ocuparam, nesse período, a segunda causa direta mais prevalente de óbito materno, diferente do observado no atual estudo. No entanto, $80,6 \%$ das mulheres eram residentes no interior do estado, e esse fato pode ter colaborado para a maior quantidade de óbitos por hemorragia, em virtude da possível peregrinação das mulheres da sua cidade de origem em busca do serviço de referência na capital. Além disso, em muitos casos, o transporte pode ter sido feito sem o suporte adequado, aumentando mais ainda a chance de morte.

Outras pesquisas realizadas no Brasil também detectaram que as causas diretas específicas de morte foram a hipertensão, a hemorragia, o aborto e as infecções puerperais ${ }^{18,19}$. A predominância dos óbitos provocados por causas diretas, por serem em grande parte evitáveis, reflete a necessidade de adequada atenção pré-natal, com realização de consultas e exames para maior conhecimento do desenvolvimento da gestação, a fim de diminuir riscos maternos e fetais associados, assim como atenção ao parto e pós-parto de qualidade. Para isso, há consenso da necessidade de formação profissional humanizada, além de educação continuada e permanente dos profissionais que atendem à mulher durante o ciclo gravídicopuerperal ${ }^{18,19}$.

Outro aspecto que merece destaque são as mortes decorrentes de aborto. Dados nacionais revelam um panorama semelhante ao encontrado neste estudo ${ }^{20}$. A estabilidade na prevalência das mortes por aborto pode ser entendida como um marcador que sinaliza a necessidade de ações efetivas para a eliminação do aborto inseguro, visto que este é classificado também como um evento evitável ${ }^{21}$. A maior parte das mulheres ainda realiza o aborto com métodos inseguros, em locais clandestinos e com profissionais sem qualificação ${ }^{22}$. Além disso, nos casos em que o aborto inseguro, é realizado com o uso do misoprostol, as doses e via de administração não são utilizadas de maneira correta, potencializando a chance de complicações ${ }^{23,24}$. No Brasil, são comuns dois atrasos que contribuem, significativamente, para o agravamento das complicações. O primeiro ocorre por dificuldade da mulher em buscar o serviço de saúde, seja pelo estigma, seja por medo de ser penalizada. O segundo, dentro dos próprios serviços, é decorrente da longa espera para o atendimento, podendo ser amplificada pelo diagnóstico e tratamento tardios das complicações do aborto por parte dos próprios profissionais de saúde ${ }^{23,25}$.

Quanto às causas hipertensivas, também se observam dificuldade e atraso na identificação precoce da doença, tanto na atenção primária durante a realização do pré-natal, como no encaminhamento/ transferência para tratamento especializado dos casos graves e correto manejo das urgências e emergências hipertensivas ${ }^{4,13}$. Dados de Campinas-São Paulo evidenciam a subutilização ou mesmo o uso de forma inadequada de medicamentos de uso consagrado no tratamento da préeclâmpsia grave e da eclâmpsia, como o sulfato de magnésio e a hidralazina ${ }^{26}$, evidenciando a falta de seguimento das diretrizes e recomendações dos órgãos nacionais e internacionais de saúde.

$\mathrm{Na}$ análise bivariada, os fatores que apresentaram associação para o óbito foram a idade, a ocupação e o número de consultas pré-natal. Houve associação entre a idade e o risco de morte materna, uma vez que as mulheres, com 30 anos ou mais, apresentaram maior chance de óbito. Em Recife-Pernambuco, foram observados elevados percentuais de mortalidade materna para mulheres na faixa etária de 20-34 anos, com aumento do risco com a idade, acentuando-se a partir dos 35 anos $^{11}$. Dados de 144 países evidenciam que as mulheres acima de 35 anos apresentaram as maiores taxas de mortalidade materna ${ }^{27}$. A idade materna avançada tem sido apontada como o fator de risco para morbidade materna, uma vez que o aumento da idade é associado à maior incidência de doenças crônicas que podem levar à morbidade e à mortalidade maternas mais elevadas ${ }^{28}$. Apesar disso, merece destaque o fato que $25 \%$ dos óbitos deste estudo foram observados entre adolescentes. No entanto, é importante ter cautela ao associar a gravidez nessa fase com fator de risco independente, sendo necessário o entendimento do fenômeno dentro de um contexto amplo das vulnerabilidades típicas dessa faixa etária ${ }^{27}$.

As mulheres que declararam não exercer uma ocupação remunerada apresentaram maior chance de morte materna no 
atual estudo. É importante esclarecer que não há concordância se a ocupação laboral durante a gravidez for um fator de risco ou de proteção $0^{5,11}$. Alguns autores sugerem que a ausência de ocupação laboral tenha intersecção com a baixa renda, e que esta última seria o fator de risco independente para a morte materna ${ }^{29}$. Por sua vez, há dados que demonstram uma situação diferente em que as mulheres com inserção produtiva apresentaram maior chance de morte relacionada à gravide $z^{12}$. Possuir uma atividade produtiva pode não representar uma situação econômica mais favorável, pois a participação no mercado de trabalho representa, para muitas mulheres, uma estratégia necessária à complementação da renda familiar. Para essas mulheres, faz-se necessário conciliar o trabalho remunerado com a realização de atividades domésticas e o cuidado dos filhos. Mais ainda, as mulheres com atividade remunerada podem procurar menos os serviços de saúde, mesmo quando ocorrer uma doença ${ }^{11}$.

Uma parcela significativa das gestantes (47,3\%) deste estudo não realizou o acompanhamento de pré-natal ou realizou um número inferior a quatro consultas, sendo esse um fator de risco para a morte materna na população estudada. Estudo de casocontrole sobre os fatores de risco para mortalidade materna em Recife-Pernambuco, entre 2001-2005, também identificou que apenas $26,2 \%$ das mulheres realizaram seis consultas ou mais ${ }^{11}$. Em 2015, apenas 40\% de todas as mulheres grávidas em países de baixa renda realizaram o número de consultas de pré-natal recomendado1. A pesquisa Nascer no Brasil, em 2012, mostrou que $60 \%$ das gestantes iniciou o pré-natal tardiamente, e que cerca de um quarto delas não realizou o número mínimo de seis consultas recomendado pelo Ministério da Saúde ${ }^{30}$. É consenso que a assistência pré-natal adequada pode contribuir para desfechos maternos mais favoráveis, ao permitir a detecção precoce e o tratamento oportuno de doenças, além de controlar fatores de risco que trazem complicações para a saúde da mãe e do concepto ${ }^{5,11,19}$.
Entre as limitações do presente estudo, destaca-se o fato de que seus resultados são baseados em dados secundários. Observouse sub-registro no preenchimento das DOs, principalmente nos campos "estado civil", "ocupação habitual" e "tipo de gravidez". Além disso, na parte IV da declaração de óbito, destinada às "causas da morte", verificaram-se dificuldade de caracterização e preenchimento adequado. Também foi possível observar, mesmo nas fichas de investigação do óbito, muitas lacunas nas informações sociodemográficas e clínicas das gestantes. Ainda mais, por se tratar de dados secundários, o estudo avaliou apenas as variáveis disponíveis nas DOs e fichas de investigação do óbito, o que pode ter dificultado a compreensão mais ampliada dos contextos em que as mortes ocorreram.

Os dados reforçam a importância da investigação sistemática do óbito materno, com o objetivo de obtenção de informações confiáveis para a melhoria da qualidade de assistência prestada à mulher no ciclo gravídico-puerperal. Esse é o primeiro estudo com metodologia caso-controle que permitiu traçar um panorama das principais causas e fatores associados ao óbito materno em Teresina. Diante do cenário apresentado, é necessária a priorização de ações de políticas públicas voltadas para a redução da mortalidade materna tendo em vista a alta RMM encontrado no município de Teresina, por causas evitáveis em sua maioria. Dessa forma, as mortes maternas poderiam ser prevenidas por meio de investimentos na qualificação dos serviços de saúde de planejamento familiar, pré-natal e assistência ao parto e puerpério. Além disso, também é imperioso garantir às mulheres uma rede de serviços de saúde com estrutura de boa qualidade, com fluxos de referências e contrarreferências satisfatórios, transporte adequado e profissionais treinados com foco para reduzir os óbitos decorrentes do abortamento inseguro e das urgências e emergências hipertensivas.

\section{REFERÊNCIAS}

1. World Health Organization. Trends in maternal mortality: 1990 to 2015. Estimates by WHO, UNICEF, UNFPA, World Bank Group and the United Nations Population Division. Geneva: WHO; 2015.

2. Alkema L, Chou D, Hogan D, Zhan S, Moller AB, Gemmill A, et al. Global, regional, and national levels and trends in maternal mortality between 1990 and 2015, with scenario-based projections to 2030: a systematic analysis by the UN Maternal Mortality Estimation Inter-Agency Group. Lancet 2016 Jan; 387(10017):462-74. doi: 10.1016/S0140-6736(15)00838-7.

3. Say L, Chou D, Gemmill A, Tunçalp O, Moller AB, Daniels J, et al. Global causes of maternal death: a WHO systematic analysis. Lancet 2014 Jun; 2(6):323-33. doi: 10.1016/S2214-109X(14)70227-X.

4. Hirshberg A, Srinivas SK. Epidemiology of maternal morbidity and mortality. Semin Perinatol. 2017 Oct; 41(6): 332-7. doi: 10.1053/j.semperi.2017.07.007.

5. St Pierre A, Zaharatos J, Goodman D, Callaghan W. Challenges and opportunities in identifying, reviewing, and preventing maternal deaths. Obstet Gynecol. 2018 Jan; 131(1):138-42. doi: 10.1097/AOG.0000000000002417.

6. Ministério da Saúde (BR). Mortalidade materna no Brasil. Bol Epidemiol
[Internet]. 2012 [acesso 2019 Set 13]; 43(1): 1-7. Disponível em: https:// portalarquivos2.saude.gov.br/images/pdf/2014/julho/23/BE-2012-43--1--pag1-a-7---Mortalidade-Materna.pdf.

7. Instituto de Pesquisa Econômica Aplicada. Objetivos de Desenvolvimento do Milênio: Relatório Nacional de Acompanhamento [Internet]. Brasília: IPEA; 2014 [acesso 2019 Se 13]. Disponível em: https://www.ipea.gov.br/portal/ images/stories/PDFs/140523_relatorioodm.pdf.

8. Ministério da Saúde (BR). Serviços de atenção materna e neonatal: segurança e qualidade. Brasília: ANVISA; 2014.

9. Szwarcwald CL, Escalante JJC, Rabello-Neto DL, Souza Jr. PRB, Victora CG. Estimation of maternal mortality rates in Brazil, 2008-2011. Cad Saude Publica 2014; 30(supl. 1): 71-83. doi: http://dx.doi.org/10.1590/0102-311X00125313.

10. Madeiro AP, Rufino AC, Lacerda, EZG, Brasil LG. Incidence and determinants of severe maternal morbidity: a transversal study in a referral hospital in Teresina, Piauí, Brazil. BMC Pregnancy Childbirth. 2015 Sep; 15: 210. doi: 10.1186/s12884-015-0648-3.

11. Leite RMB, Araújo TVB, Albuquerque RM, Andrade ARS, Duarte NPJ. Fatores 


\section{Mortalidade materna em Teresina}

de risco para mortalidade materna em área urbana do Nordeste do Brasil. Cad Saude Publica 2011; 27(10): 1977-1985. doi: https://doi.org/10.1590/S0102$311 \times 2011001000011$.

12. Resende LV, Rodrigues RN, Fonseca MC. Mortes maternas em Belo Horizonte, Brasil: percepções sobre qualidade da assistência e evitabilidade. Rev Panam Saude Publica, 2015; 37(4-5):218-24.

13. Burton GJ, Redman CW, Roberts JM, Moffett A. Pre-eclampsia: pathophysiology and clinical implications. BMJ. 2019 Jul; 366: I2381. doi: 10.1136/bmj.I2381.

14. Vega CEP, Soares VMN, Lourenço Francisco Nasr AM. Mortalidade materna tardia: comparação de dois comitês de mortalidade materna no Brasil. Cad Saude Publica 2017; 33(3): e00197315. doi: https://doi.org/10.1590/0102$311 \times 00197315$.

15. Mota SMM, Gama SGN, Theme FMM. Mortalidade materna no município de Belém, estado do Pará, em 2004: uma avaliação do Sistema de Informações sobre Mortalidade. Epidemiol Serv Saude. 2008 Mar; 17(1): 33-42. doi: http:// dx.doi.org/10.5123/S1679-49742008000100004.

16. Martins ACS, Silva LS. Perfil epidemiológico de mortalidade materna. Rev Bras Enferm. 2018; 71(Suppl 1): 677-83. doi: https://doi.org/10.1590/00347167-2017-0624.

17. Madeiro AP, Rufino AC, Nunes MD, Queiroz IC, Carvalho KR, Queiroz LC. Mortalidade de mulheres em idade fértil no Piauí, Brasil, 2008-2012: causas básicas dos óbitos e fatores associados. Rev Epidemiol Control Infec. 2018 OutDez; 8(4): 442-9.

18. Souza JP. Mortalidade materna e desenvolvimento: a transição obstétrica no Brasil. Rev Bras Ginecol Obstet. 2013 Dez; 35(12):533-5. doi: https://doi. org/10.1590/S0100-72032013001200001.

19. Pacagnella RC, Nakamura-Pereira M, Gomes-Sponholz F, Aguiar RALP, Guerra GVQL, Diniz CSG, et al. Maternal mortality in Brazil: proposals and strategies for its reduction. Rev Bras Ginecol Obstet. 2018 Sep; 40(9): 501-6. doi: https://doi. org/10.1055/s-0038-1672181.

20. Pícoli RP, Cazola LHO, Lemos EF. Mortalidade materna segundo raça/cor, em Mato Grosso do Sul, Brasil, de 2010 a 2015. Rev Bras Saude Mater Infant. 2017; 17(4): 729-37. doi: https://doi.org/10.1590/1806-93042017000400007.

21. Darney PD, Nakamura-Pereira M, Regan L, Serur L, Thapa K. Maternal mortality in the United States compared with Ethiopia, Nepal, Brazil, and the
United Kingdom: contrasts in reproductive health policies. Obstet Gynecol. 2020 Jun;135(6):1362-6. doi: 10.1097/AOG.0000000000003870.

22. Ganatra B, Gerdts C, Rossier C, Johnson BR, Tunçalp O, Assifi A, et al. Global, regional, and subregional classification of abortions by safety, 2010-14: estimates from a Bayesian hierarchical model. Lancet. 2017 Nov; 390(10110): 2372-2381. doi: 10.1016/S0140-6736(17)31794-4.

23. Nunes MD, Madeiro AP, Diniz D. Histórias de aborto provocado entre adolescentes em Teresina, Piauí, Brasil. Cien Saude Colet. 2013;18(8): 2311-8. doi: https://doi.org/10.1590/S1413-81232013000800015.

24. Calvert C, Owolabi OO, Yeung F, Pittrof R, Ganatra B, Tunçalp Ö, et al. The magnitude and severity of abortion-related morbidity in settings with limited access to abortion services: a systematic review and meta-regression. BMJ Glob Health. 2018 Jun; 3(3): e000692. doi: 10.1136/bmjgh-2017-000692.

25. Madeiro AP, Rufino AC. Maus-tratos e discriminação na assistência ao aborto provocado: a percepção das mulheres em Teresina, Piauí, Brasil. Cien Saude Colet. 2017 Ago; 22(8): 2771-80. doi: https://doi.org/10.1590/141381232017228.04252016 .

26. Lotufo FA, Parpinelli MA, Osis MJ, Surita FG, Costa ML, Cecatti JG. Situational analysis of facilitators and barriers to availability and utilization of magnesium sulfate for eclampsia and preeclampsia in the public health system in Brazil. BMC Pregnancy Childbirth. 2016 Aug; 16(1): 254. doi: 10.1186/s12884-0161055-0.

27. Nove A, Matthews Z, Neal S, Camacho AV. Maternal mortality in adolescents compared with women of other ages: evidence from 144 countries. Lancet Glob Health. 2014 Mar; 2(3): e155-64. doi: 10.1016/S2214-109X(13)70179-7.

28. Davis NL, Hoyert DL, Goodman DA, Hirai AH, Callaghan WM. Contribution of maternal age and pregnancy checkbox on maternal mortality rates in the United States, 1978-2012. Am J Obstet Gynecol. 2017 Sep: 217(3): 352.e1-352. e7. doi: 10.1016/j.ajog.2017.04.042.

29. Small MJ, Allen TK, Brown HL. Global disparities in maternal morbidity and mortality. Semin Perinatol. 2017 Aug; 41(5): 318-22. doi: 10.1053/j. semperi.2017.04.009.

30. Martinelli KG, Santos NET, Gama SGN, Oliveira AE. Access to prenatal care: inequalities in a region with high maternal mortality in southeastern Brazil. Cien Saude Colet 2016 May; 21(5): 1647-58. http://dx.doi.org/10.1590/141381232015215.23222015.

\section{Como citar este artigo/How to cite this article:}

Timóteo NLS, Rufino AC, Madeiro A. Mortalidade materna em Teresina, Piauí, Brasil: um estudo caso-controle. J Health Biol Sci. 2021; 9(1):1-9. 